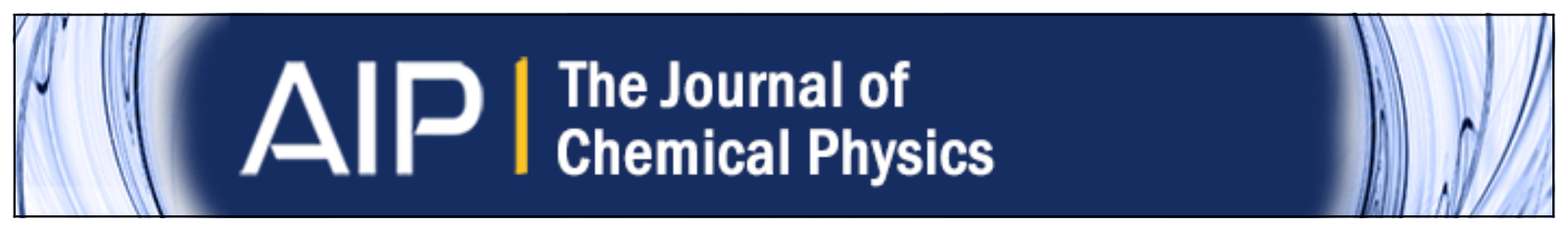

Effects of the Gaussian energy dispersion on the statistics of polarons and bipolarons in conducting polymers

Juan Bisquert, Germà Garcia-Belmonte, and Jorge Garca-Cañadas

Citation: The Journal of Chemical Physics 120, 6726 (2004); doi: 10.1063/1.1665781

View online: http://dx.doi.org/10.1063/1.1665781

View Table of Contents: http://scitation.aip.org/content/aip/journal/jcp/120/14?ver=pdfcov

Published by the AIP Publishing

AlP Re-register for Table of Content Alerts 


\title{
Effects of the Gaussian energy dispersion on the statistics of polarons and bipolarons in conducting polymers
}

\author{
Juan Bisquert, ${ }^{\text {a) }}$ Germà Garcia-Belmonte, and Jorge García-Cañadas \\ Departament de Ciències Experimentals, Universitat Jaume I, 12080 Castelló, Spain
}

(Received 17 November 2003; accepted 13 January 2004)

\begin{abstract}
We discuss the interpretation of usually broad oxidation peaks observed in electronically conducting polymers, in terms of the statistical distributions functions of polarons and bipolarons. The analysis is based on examining the chemical capacitance, that relates the change of concentration to a modification of the chemical potential of a given species, for different statistical models. We first review the standard models for single energy species that provide a nernstian dependence, and the limitations of these models are discussed. A new model that assumes a Gaussian distribution of energies related to molecular geometry fluctuations is suggested, and this model shows excellent agreement with the results of electrochemical oxidation of polypyrrole in quasiequilibrium conditions. From a fit of the data, it is found that the density of conjugated chain segments in polypyrrole, $N_{s} \approx 10^{21} \mathrm{~cm}^{-3}$, shows a Gaussian distribution of half width $\sigma \approx 170 \mathrm{meV}$, tentatively attributed to bipolaron formation energies. (C) 2004 American Institute of Physics.
\end{abstract}

[DOI: $10.1063 / 1.1665781]$

\section{INTRODUCTION}

Electronically conducting polymers can be doped and undoped electrochemically. A shift of the potential to positive values changes the film from electrically insulating to a conducting state. Physically the mechanism is a change of electron occupancy in the conjugated chains that creates holes which transport electrical current as polarons. Electrochemically, this process can be thought of as an oxidation of the polymer. The converse process is a shift of the potential to negative values that fills the holes, i.e., a reduction of the film that turns it to the electrically insulating (nonconducting) state.

The potential that is monitored and varied in electrochemical and spectroelectrochemical measurements of electronically conducting polymers, $U$, is related to the electrochemical potential of electrons in the film, $\mu$, as $U=-(\mu$ $\left.-\mu_{\text {ref }}\right) / e$, with respect to the electrochemical potential in the reference electrode $\mu_{\text {ref }}$, which can be assumed constant. Here $e$ stands for the positive elementary charge. If the polymer film remains close to equilibrium during measurement, the oxidation process is described by the thermodynamic function $\mu(n)$, where $n$ is the concentration of electrons in the polymer. It is convenient to express the electrochemical potential of electrons as $\mu(p)$, where $p$ is the density of holes (extracted electrons) in the form of polarons $(P)$ and bipolarons $(B)$, taking into account that $n+p=N_{\pi}$, the density of $\pi$ orbitals.

Theoretically, the electrochemical potential (or Fermi level) $\mu(p)$, as a function of concentration, is derived from assumptions on the distribution and interaction of $P$ and $B$ species. ${ }^{1-5}$ The simplest model for a single energy, noninteracting species gives the Langmuir isotherm, the Nernst equa-

\footnotetext{
a) Author to whom correspondence should be addressed. Electronic mail: bisquert@uji.es
}

tion, and the Fermi-Dirac statistics. Independently of the method used to derive the result, these are all equivalent expressions for the chemical potential based on the multiplicity of configurations (entropy) for $p$ polarons distributed in $N$ sites per unit volume. Interactions are often treated in the mean-field (Bragg-Williams) approximation corresponding to the Frumkin isotherm. ${ }^{6}$ Either form, Langmuir or Frumkin, for the chemical potential, is based on the assumption that all sites have the same energy. This assumption is suitable for many systems, e.g., adsorption in crystalline surfaces or intercalation in crystalline bulk materials, provided that the different kinds of sites in the unit cell are well separated in energy. In contrast, the amorphous materials, such as glasses, are characterized by a lack of spatial periodicity. This causes a wide variation of the local environment of electrons in the material, implying a wide distribution of classes of sites. Therefore the entropy properties of the electron distribution are expected to depart markedly from single site distributions such as Langmuir form. This idea has been realized in a number of amorphous and disordered materials: The configurational entropy in glass-forming liquids, ${ }^{7}$ the electron distribution in nanostructured $\mathrm{TiO}_{2}$ electrodes ${ }^{8}$ and the intercalation of $\mathrm{Li}$ in amorphous $\mathrm{WO}_{3} .{ }^{9}$

Since the electrochemically prepared conducting polymers are usually far from crystalline structure, it should be reasonable to expect a wide distribution of the sites for $P$ and $B$. In particular, the Gaussian distribution has been found quite useful for the glass transition to the vitreous state, ${ }^{7}$ for ion transport in ion-conducting glasses, ${ }^{10}$ and for intercalation in amorphous materials. ${ }^{9}$ In the case of organic conducting polymers, disorder due to variation in molecular surroundings is often described by a Gaussian distribution of site energies (diagonal disorder) that is an important factor in the transport of electrons in these materials. ${ }^{11,12}$ It is natural to develop the model of uncorrelated dispersion of site ener- 
gies (Gaussian distribution) for the central relationship governing electrochemical doping (oxidation) of conducting polymers $\mu(p)$, and that is the aim of the present paper.

We will also compare the model with the experimental results of electrochemical doping (oxidation) of polypyrrole. Experimentally, usually the current is monitored as the potential varies at a constant speed, $s=d U / d t$ (cyclic voltammetry, $\mathrm{CV}$, technique). The electronic current flowing into a unit volume of the polymer is

$$
j=-e \frac{d n}{d t}=-e \frac{d n}{d \mu} \frac{d \mu}{d t}=s e^{2} \frac{d n}{d \mu}=-s e^{2} \frac{d p}{d \mu} .
$$

Therefore the results of $\mathrm{CV}, j(U)$, are related directly to the reciprocal of the derivative of the thermodynamic function $\mu(p)$. It is useful to introduce the definition of the electrochemical capacitance ${ }^{13}$ (the "redox capacity" in the specific language of conducting polymers ${ }^{1}$ ),

$$
C_{\mu}=e^{2} \frac{d n}{d \mu}=-e^{2} \frac{d p}{d \mu},
$$

so that voltammetry results can be expressed succinctly as

$$
j={ }_{s} C_{\mu} .
$$

Hence $C_{\mu}(\mu)$ is measured directly by cyclic voltammetry. From Eq. (3), quasiequilibrium conditions in CV can be confirmed by an invariant $j(U) / s$ at different speeds. ${ }^{14}$

In conducting polymers there occurs undoubtedly a change of Fermi level with respect to electronic energy levels, so that a substantial component of the electrochemical capacitance $C_{\mu}$ is the chemical capacitance related to a variation of the chemical potential with respect to a change of concentration of polarons. ${ }^{15}$ The chemical capacitance is controlled by a change of entropy with the concentration. It is characterized by a strong dependence on the potential, which is some ranges of composition takes the exponential form. While this form is broadly described by many models, the precise type of dependence $C_{\mu}(U)$ will be considered a critical test for the statistical model. Indeed the present research was motivated by persistent observation of slopes different than a multiple of $60 \mathrm{mV} /$ decade (the nernstian form) in electrochemical measurements of conducting polymers.

The voltammetry of conducting polymers including oxidation and reduction peaks shows a number of general features that are well described in the literature. ${ }^{3,4,16,17}$ Beyond the oxidation peak the voltammetry shows a current plateau and the subsequent reduction peak is broader and displaced from the oxidation peak. The interpretation of these features has been debated, see Refs. 3 and 4 for a summary. In this paper, we are mainly interested in the experimental aspects of conducting polymers that can be described by molecularstatistical models corresponding to thermodynamic equilibrium. We consider the quasiequilibrium part of the oxidation peak and we show that the model based on the Gaussian distribution of energies provides an excellent description of the changes in the polymer while it is doped electrochemically. Our approach changes the interpretation of the oxidation peak. In nernstian (or Langmuir) model the peak is related to $\frac{1}{2}$ occupancy of a single class of site. In contrast, in the Gaussian distribution of energies the shape of the peak is determined by the shape of the distribution. This new insight is expected to provide a key step in the construction of a more general model including hysteresis effects in the potential range beyond the oxidation peak, but this is left for future investigations.

In the remaining part of this paper, we first present theoretical models and then analyze experimental results on $\mathrm{CV}$ of polypyrrole. In the theoretical sections we study generally the properties of the chemical capacitance in relation to the assumptions on statistical distributions for polarons and bipolarons in the conducting polymers. This theoretical study aims to show the different implications of the Gaussian distribution, not only for the $\mathrm{CV}$ measurements discussed later on (in which it is difficult to separate contributions of $P$ and $B$ to the oxidation peak), but also for ESR spectroelectrochemistry (changing progressively the film potential) that may be attempted in the future. In Sec. II we review a number of statistical models proposed in the literature in order to show that single energy (nernstian) models place strong constraints on the dependence of the chemical capacitance on potential. In Sec. III we present the derivation of the new model with the Gaussian distribution of sites. Section IV describes the experimental procedures and Sec. V presents a discussion of the results and the comparison of the different models. We finish with the conclusions in Sec. VI.

\section{NERNSTIAN MODELS FOR CONDUCTING POLYMERS}

The polymer is considered as a collection of $N_{s}$ conjugated chain segments per unit volume, each containing $m$ monomers. These segments describe the extension of polarons or bipolarons in the polymer chain, so that each segment contains at most one charged excitation $(P$ or $B)$. Taking the energy reference at the center of the gap of width $2 \varepsilon$, the energy for adding an electron is $\varepsilon_{c}=\varepsilon$, and the energy for extracting an electron (adding a hole to the valence band) is $\varepsilon_{\nu}=-\varepsilon$. When a hole is added to the polymer, relaxation of the atomic positions will occur extremelly rapidly. The energy of the polaron formation, $-\varepsilon_{P}$, is lower than that of the unrelaxed hole, $\varepsilon_{\nu}$. Further, a double charged excitation, the bipolaron, can be formed in the conjugated chain segment ${ }^{18,19}$ with a total energy of formation $-\varepsilon_{B}$. The intrinsic degeneracies of the polaronic states ${ }^{3,20}$ will be neglected in order to emphasize the dependencies on potential.

If only $P$ can be formed, their density is given by

$$
n_{P}=N_{s} f_{P}\left(\mu, \varepsilon_{P}\right),
$$

where $\mu$ is the electrochemical potential of electrons, and the distribution function $f_{P}$ is the Fermi-Dirac function for holes, shown in Fig. 1(a)

$$
f_{P}\left(\mu, \varepsilon_{P}\right)=\frac{1}{1+e^{\left(\varepsilon_{P}+\mu\right) / k_{B} T}} .
$$

The chemical capacitance of polarons,

$$
C_{\mu}=-N_{s} e^{2} \frac{d f_{P}}{d \mu},
$$

has the value 


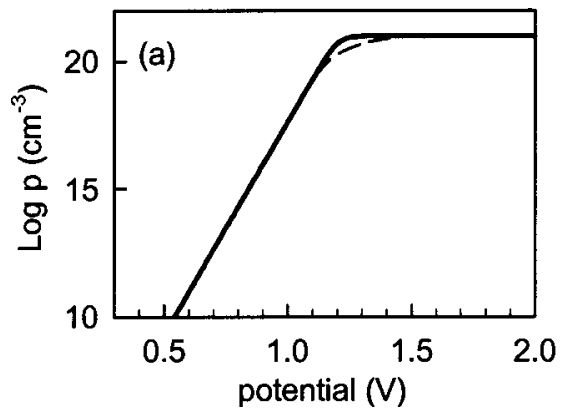

$$
C_{\mu}=\frac{N_{s} e^{2}}{k_{B} T} f_{P}\left(1-f_{P}\right) .
$$

This is the standard form of the "redox capacitance" for a nernstian species, ${ }^{1}$ Fig. 1(b). It forms a peak at the standard potential $\left[f_{P}\left(\mu_{0}, \varepsilon_{P}\right)=\frac{1}{2}\right]$, with a slope of $60 \mathrm{mV} /$ decade at the cathodic side.

Note that Eq. (5) is the Langmuir isotherm and takes also the form of the Nernst expression,

$$
\mu=-\varepsilon-k_{B} T \ln \frac{n_{P}}{N_{s}-n_{P}} .
$$

Several variants of Eq. (8) have been described in the literature. Interactions are often treated with the mean-field (Bragg-Williams) approximation corresponding to the Frumkin isotherm. ${ }^{6}$ In this model the local interactions are replaced by an averaged field (molecular field) which is determined by the distribution of electrons and which in turn governs the distribution itself. ${ }^{21}$ The result is

$$
\mu=-\varepsilon-k_{B} T \ln \frac{n_{P}}{N_{\nu}-n_{P}}-b k_{B} T n_{P},
$$

where $b$ is the dimensionless interaction parameter, which typically has a value $\approx 4$, for an interaction energy of 100 meV per particle. Exactly the same expression, Eq. (9), is obtained on the assumption of energy shift by charging the polymer/solution interface. ${ }^{1}$ In both models, molecular field interaction and band unpinning, the energies of all polaron levels shift equally. For reasonable values of $b$ the interaction term of Eq. (9) becomes significant only at very high concentration and does not modify the nernstian slope of the chemical capacitance, as shown in Fig. 1(b), for a relatively large interaction constant $b=8$.

Other approaches consider the contribution of additional species to the chemical potential, besides electronic ones. For electrons and ions, maintaining charge neutrallity, the combination of two similar contributions to the electrochemical potential gives a variation of $120 \mathrm{mV}$ in the chemical capacitance. ${ }^{1}$ By taking $\alpha$ segments as a unit of mechanical relaxation, the nernstian slope takes the value $\alpha 60 \mathrm{mV}^{5}$

Many studies based on simultaneous voltammetry and spin relaxation (ESR) have demonstrated that the oxidation of conducting polymers involves both $P$ and $B$ with densities $n_{P}$ and $n_{B} \cdot{ }^{16,22,23}$ In this case there will be chain segments either empty or singly or doubly occupied by holes among the available number $N_{s}$. The free energy takes the form

$$
F=\left(\varepsilon_{P}+\mu\right) n_{P}+\left(\varepsilon_{B}+2 \mu\right) n_{B}-k_{B} T \ln \Omega,
$$

FIG. 1. (a) Density of polarons, $n_{P}$, for a density of conjugated chain segments $N_{s}=10^{21} \mathrm{~cm}^{-3}$, with energy $\varepsilon_{P}=1.2 \mathrm{eV}$, in the absence of formation of bipolarons. (b) The chemical capacitance per unit volume. The thin lines show the same system with a mean-field repulsive interaction that contributes a term $8 k_{B} T n_{P}^{2} / 2$ to the free energy.

where the number of arrangements is given by

$$
\Omega=\frac{N_{s} !}{\left(N_{s}-n_{P}-n_{B}\right) ! n_{P} ! n_{B} !}
$$

and using the Stirling approximation one obtains

$$
\begin{aligned}
F= & \left(\varepsilon_{P}+\mu\right) n_{P}+\left(\varepsilon_{B}+2 \mu\right) n_{B} \\
& +k_{B} T\left(n_{P} \ln \frac{n_{P}}{n_{0}}+n_{B} \ln \frac{n_{B}}{n_{0}}-N_{s} \ln \frac{N_{s}}{n_{0}}\right) .
\end{aligned}
$$

The result of minimizing $F$ gives the expressions ${ }^{22}$

$$
\begin{aligned}
& -\mu=\varepsilon_{P}+k_{B} T \ln \frac{n_{P}}{n_{0}}, \\
& -\mu=\varepsilon_{B}-\varepsilon_{P}+k_{B} T \ln \frac{n_{B}}{n_{P}} .
\end{aligned}
$$

Equations (13) and (14) correspond to equilibrium ratios of the reactions $A \leftrightarrow P^{+}+e^{-}$and $P^{+} \leftrightarrow B^{++}+e^{-}$where $A$ is a neutral segment, with density $n_{0}=N_{s}-n_{P}-n_{B}$. Equations (13) and (14) provide the distribution functions, ${ }^{3,20}$

$$
\begin{aligned}
& f_{P}\left(\mu, \varepsilon_{P}, \varepsilon_{B}\right)=\frac{1}{1+e^{\left(\varepsilon_{P}+\mu\right) / k_{B} T}+e^{-\left(\varepsilon_{B}-\varepsilon_{B}+\mu\right) / k_{B} T}} \\
& f_{B}\left(\mu, \varepsilon_{P}, \varepsilon_{B}\right)=\frac{1}{1+e^{2\left(\varepsilon_{B} / 2+\mu\right) / k_{B} T}+e^{\left(\varepsilon_{B}-\varepsilon_{B}+\mu\right) / k_{B} T}} .
\end{aligned}
$$

The densities of $P$ and $B$ are given by

$$
\begin{aligned}
& n_{P}=N_{s} f_{P}\left(\mu, \varepsilon_{P}, \varepsilon_{B}\right), \\
& n_{B}=N_{s} f_{B}\left(\mu, \varepsilon_{P}, \varepsilon_{B}\right) .
\end{aligned}
$$

The densities of $P$ and $B$ are equal, $n_{P}\left(\mu_{2}\right)=n_{B}\left(\mu_{2}\right)$, at the potential

$$
\mu_{2}=-\varepsilon_{B}+\varepsilon_{P} .
$$

Further, $n_{P}\left(\mu_{1}\right)=n_{0}\left(\mu_{1}\right)$ at the potential

$\mu_{1}=-\varepsilon_{P}$.

Chemical capacitances are given by the expressions

$$
\begin{aligned}
& C_{\mu}^{P}=-N_{s} e^{2} \frac{d f_{P}}{d \mu}\left(\mu, \varepsilon_{P}, \varepsilon_{B}\right), \\
& C_{\mu}^{B}=-2 N_{s} e^{2} \frac{d f_{B}}{d \mu}\left(\mu, \varepsilon_{P}, \varepsilon_{B}\right),
\end{aligned}
$$



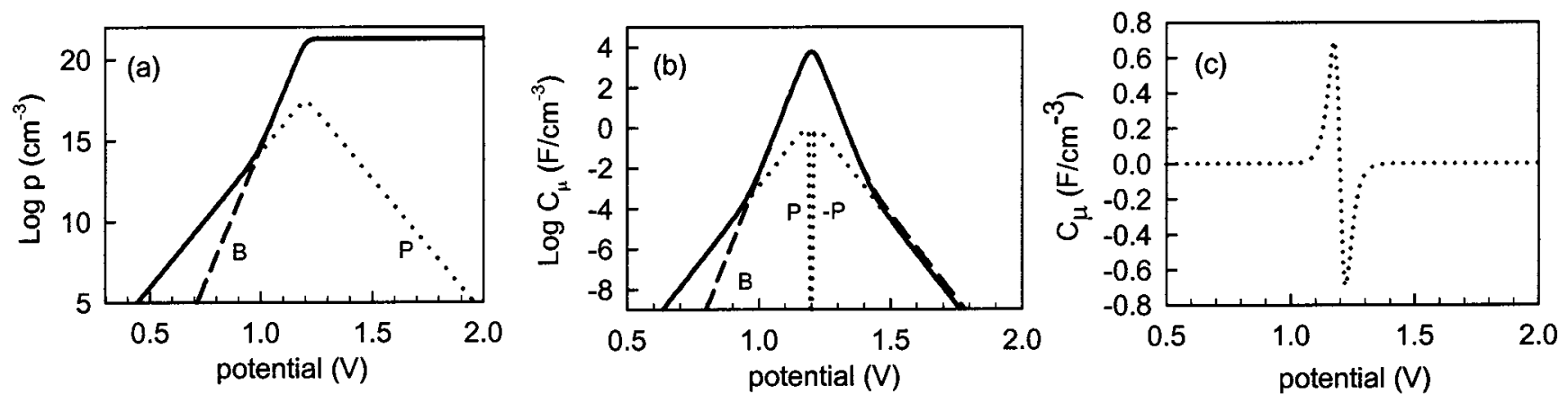

FIG. 2. (a) Densities of polarons, $n_{P}$, holes in bipolarons, $2 n_{B}$, and total holes, $n_{P}+2 n_{B}$ (thick line), for a density of conjugated chain segments $N_{s}$ $=10^{21} \mathrm{~cm}^{-3}$, with energies $\varepsilon_{P}=1.4 \mathrm{eV}$ and $\varepsilon_{B}=2.4 \mathrm{eV}$, as a function of potential, and (b) the corresponding partial and total chemical capacitances per unit volume, in logarithmic scale. The line $(-P)$ is a negative capacitance contribution of polarons. (c) The partial capacitance of polarons in linear scale.

which can be calculated readily from Eqs. (15) and (16), but are not written here explicitly.

The results of the $P-B$ model with single formation energies (in opposition to the distribution considered later on) are illustrated in Fig. 2 for the case in which $B$ are energetically favored $\left(\varepsilon_{B}<2 \varepsilon_{P}\right)$. Initially the oxidation process is dominated by $P$ 's Fig. 2(a), but $B$ 's become predominant at potential $\mu_{1}$, and the concentration of $B$ stabilizes after $\mu_{3}$ $=\varepsilon_{P}-\varepsilon_{B} / 2$ (where $\left.f_{B} \approx \frac{1}{2}\right)$. It is interesting to note that the density of $P$ shows a maximum at $\mu_{3}$ and decreases monotonically thereafter. ${ }^{3}$ This behavior is reported in ESR measurements. ${ }^{16,22,23}$

The chemical capacitance, shown in Fig. 2(b), shows first a $60-\mathrm{mV}$ and then $30-\mathrm{mV}$ decade variation, corresponding to the regions dominated by formation of $P$ and $B$, respectively. Then a peak centered in $\mu_{3}$ is observed. It is important to recognize the peculiar behavior of the partial chemical capacitance of the polarons, Fig. 2(c). This capacitance switches suddenly to a negative value at the potential $\mu_{3}$. Similar features occur when the $P$ are more stable than $B$, Fig. 3. This negative capacitance feature occurs because the density of polarons is decreasing even though their own driving force for formation is becoming larger, i.e., the Fermi level $\mu$ is moving further below the energy $-\varepsilon_{P}$. Of course the emptying process of the polaron level is related to an even larger driving force for $B$ formation by recombination of $P$ pairs.

In general, the chemical capacitance is related to stability conditions of the system. From statistical thermodynamics, the following relationship exists between the fluctuation of the number density of particles $x$ in an open system of volume $V$, and the variation of the mean value, $\langle x\rangle$, with respect to chemical potential $\mu_{x}$,

$$
\left\langle(\delta x)^{2}\right\rangle=\left\langle x^{2}\right\rangle-(\langle x\rangle)^{2}=\frac{k_{B} T}{V} \frac{\partial\langle x\rangle}{\partial \mu_{x}} .
$$

Therefore the relationship between mean fluctuation of density of species $x$ and its chemical capacitance is

$$
\left\langle(\delta x)^{2}\right\rangle=\frac{k_{B} T}{V e^{2}} C_{\mu}^{x} .
$$

When $C_{\mu}$ and $\left\langle(\delta x)^{2}\right\rangle$ are positive, density fluctuations larger than $\left\langle(\delta x)^{2}\right\rangle^{1 / 2}$ have a low probability and the system is stable. ${ }^{24}$ But if $C_{\mu}=0$ and negative values, the system is thermodynamically unstable and may separate into different phases. The negative chemical capacitance indicates that $P$ may not follow the random distribution assumed in Eq. (11), but could tend to aggregate and form regions of different density. It is interesting to note that some recent detailed ESR spectroelectrochemistry results ${ }^{25}$ on poly(3,4ethylenedioxythiophene) (PEDOT) show a stabilization of the number of spins when the potential is scanned positively beyond the peak, and a certain hysteresis on return in the cathodic direction. In fact, it is well known that hysteresis in $\mathrm{CV}$ occurs only when the potential is scanned at more positive values than the maximum of the oxidation peak. Further discussion of these questions will be presented in a separate report.
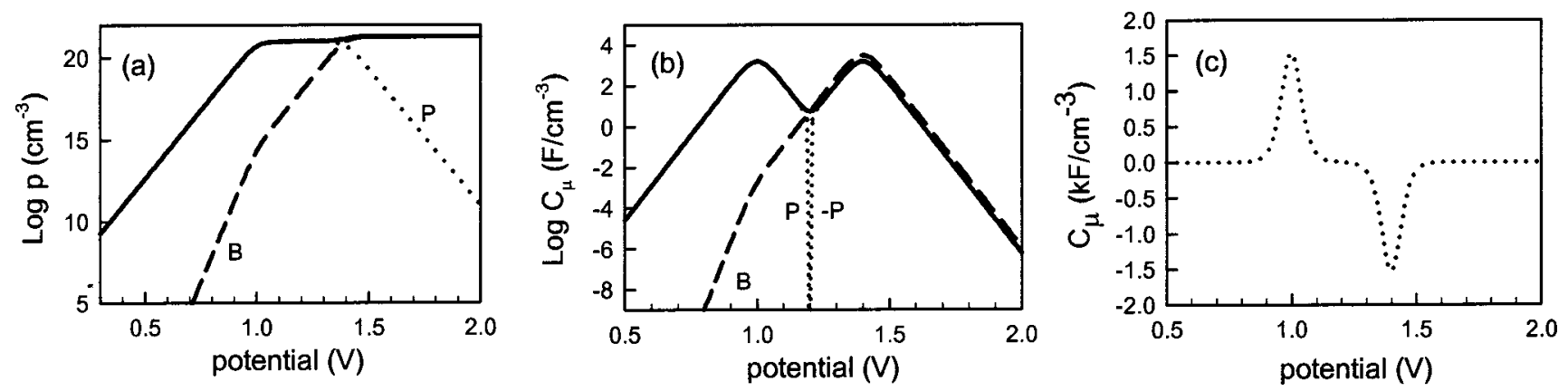

FIG. 3. Same as Fig. 2, with energies $\varepsilon_{P}=1.0 \mathrm{eV}$ and $\varepsilon_{B}=2.4 \mathrm{eV}$. 

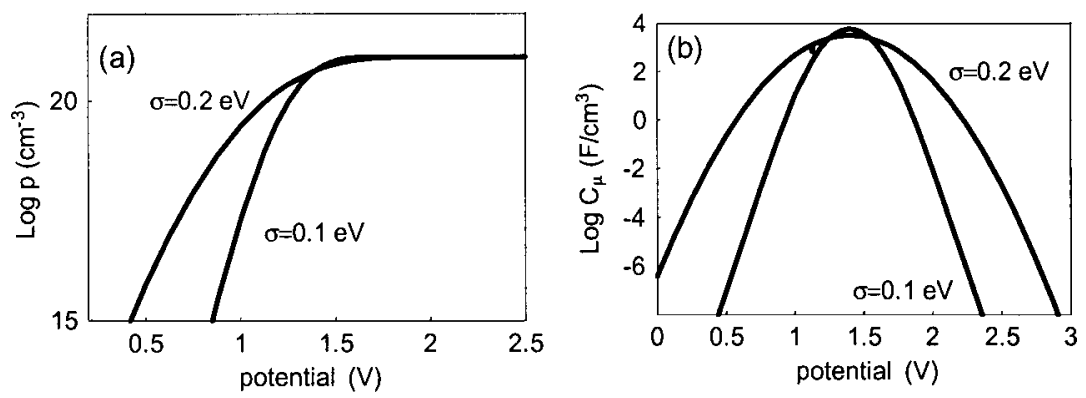

FIG. 4. (a) Densities of polarons, $n_{P}$, as a function of potential, for a density of conjugated chain segments $N_{s}=10^{21} \mathrm{~cm}^{-3}$. The energies of $P$ have a Gaussian distribution with mean values $\varepsilon_{P}^{0}=1.4 \mathrm{eV}$ and variances $\sigma_{P}$ as indicated. (b) The corresponding chemical capacitances, per unit volume in logarithmic scale.
In conclusion to this section, all the models reviewed have in common that the formation of polarons is governed by the Fermi level crossing a well defined energy level in the gap. This leads to basically Boltzmann-Nernst dependence in the chemical capacitance, which forms asymptotically a straight line (in semilog representation) of slope $30 \mathrm{mV}$ for bipolarons, $60 \mathrm{mV}$ for polarons, $120 \mathrm{mV}$ for polarons and ions, etc.

\section{GAUSSIAN DISTRIBUTION OF SITE ENERGIES}

In spite of the widespread use of the single energy models outlined in the previous section in electrochemistry measurements, it is also widely accepted that the energies of $P$ and $B$ states in the band gap form a wide distribution. This was first infered from the broadening of the absorption spectra. In polypyrrole, bands of $\approx 0.4 \mathrm{eV}$ width where ascribed to overlap between bipolaron states. ${ }^{19}$ In general, disorder or structural correlations with correlation lengths of a few intermolecular distances lead to a dispersion of energies in the $P$ and $B$ states. If the distribution is viewed as the sum of variations of internal coordinates whose properties are uncorrelated, then the central limit theorem suggests a Gaussian distribution. More specifically it was shown that the Gaussian distribution in conducting polymers is caused by the fluctuation of the lattice polarization energies, ${ }^{12}$ dipole interactions, ${ }^{26}$ and molecular geometry fluctuations. ${ }^{27}$

It is possible to give an a priori estimation of the width of the distribution. ${ }^{12}$ Absorption (and fluorescent) bands of disordered organic solids are usually of Gaussian shape with a standard deviation of typically $60 \mathrm{meV}$. Comparing the interactions with the surrounding lattice between an exciton and a charge carrier it is demonstrated that the width of the density of states for charge carriers is about 1.5 times as big as that for singlet excitation. Hence the width should be of order $100 \mathrm{meV}$. In addition, optical absorption spectroscopy and spectral dispersion measurements show that the neutral exciton excited states have a Gaussian energy distribution. ${ }^{28}$

For only one species $(P)$ we consider that the $N_{s}$ chain segments per unit volume are distributed in energy according to the Gaussian expression with mean energy $\varepsilon_{P}^{0}$ and half width $\sigma_{P}$,

$$
g(\varepsilon)=\frac{N_{s}}{\sqrt{2 \pi} \sigma_{P}} \exp \left[-\frac{\left(\varepsilon-\varepsilon_{P}^{0}\right)^{2}}{2 \sigma_{P}^{2}}\right] .
$$

The derivation of the statistical function assumes a random distribution of the $P$ among the available levels, the number of sites for each level $\varepsilon$ being given by Eq. (25). The total number of arrangements $\Omega$ is the product of the number of arrangements for each level. A straightforward calculation shows that the result of minimizing the free energy is given by Eq. (5), $f(\mu, \varepsilon)$, for the energy level $\varepsilon$. The total number of polarons, as a function of the electrochemical potential, is obtained by integration

$$
n_{P}=\int_{-\infty}^{+\infty} g(\varepsilon) f_{P}(\mu, \varepsilon) d \varepsilon
$$

The chemical capacitance is given by

$$
C_{\mu}=-e^{2} \int_{-\infty}^{+\infty} g(\varepsilon) \frac{d f_{P}}{d \mu}(\mu, \varepsilon) d \varepsilon .
$$

Observing in Eq. (5) that $d f_{P} / d \mu=d f_{P} / d \varepsilon$, integrating Eq. (27) by parts, and using the zero-temperature limit of the Fermi distribution, Eq. (27) can be expressed as
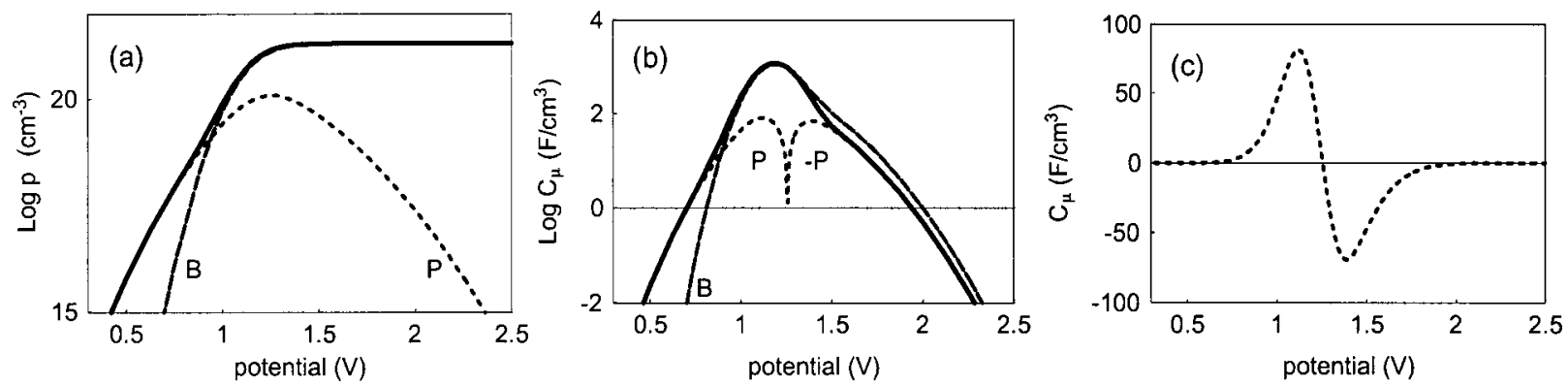

FIG. 5. (a) Densities of polarons, $n_{P}$, holes in bipolarons, $2 n_{B}$, and holes, $n_{P}+2 n_{B}$ (thick line), as a function of potential, for a density of conjugated chain segments $N_{s}=10^{21} \mathrm{~cm}^{-3}$. The energies of both $P$ and $B$ have a Gaussian distribution with mean values $\varepsilon_{P}^{0}=1.4 \mathrm{eV}$ and $\varepsilon_{B}^{0}=2.4 \mathrm{eV}$ and variances $\sigma_{P}$ $=0.1 \mathrm{eV}, \sigma_{B}=0.2 \mathrm{eV}$. (b) The corresponding partial and total chemical capacitances, per unit volume in logarithmic scale. The line $(-P)$ is a negative capacitance contribution of polarons. (c) The partial capacitance of polarons in linear scale. 

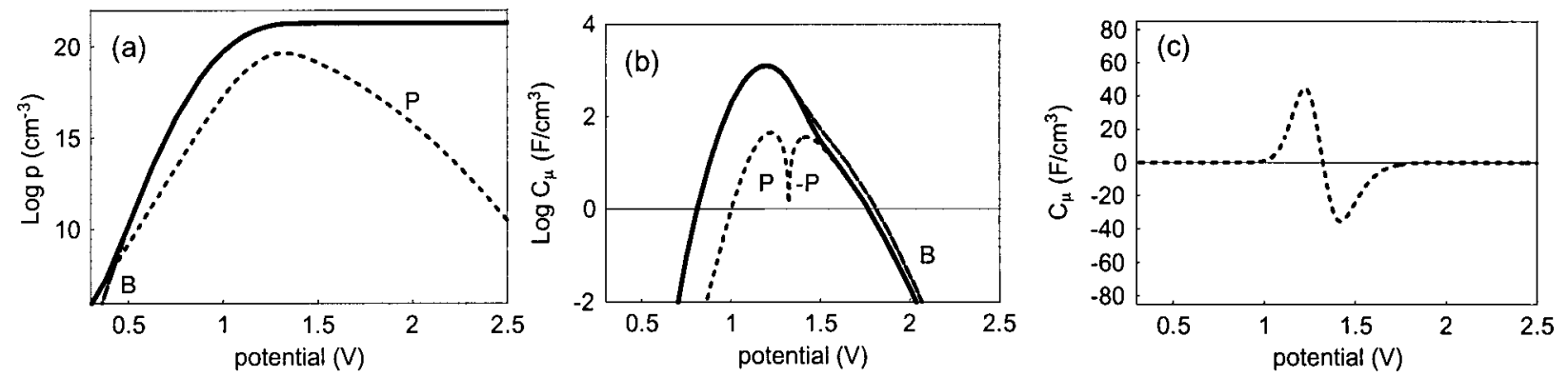

FIG. 6. Same as Fig. 5 for a Gaussian distribution of $P$ and $B$ with mean values $\varepsilon_{P}^{0}=1.4 \mathrm{eV}$ and $\varepsilon_{B}^{0}=2.4 \mathrm{eV}$ and variances $\sigma_{P}=\sigma_{B}=0.2 \mathrm{eV}$.

$$
C_{\mu}=e^{2} g(-\mu),
$$

so that the chemical capacitance as a function of potential corresponds to the density-of-states function at the energy level $\varepsilon=-\mu$. This shows that the shape of the CV peak relates directly to the shape of the distribution.

These results are illustrated in Fig. 4. The main contrast with respect to the previous models is that the chemical capacitance vs potential does not form a straight asymptotic line in the semilog representation. Indeed, the chemical capacitance is a parabolla, Eqs. (25) and (28), so that $\ln C_{\mu}$ shows straight wings when plotted versus $\left(\mu+\varepsilon_{P}^{0}\right)^{2}$. We remark also the very broad oxidation peaks that are obtained for characteristic values of the half width of the distribution.

This approach can be readily extended to the case in which both $P$ and $B$ can be formed upon oxidation. If we describe each species with its own characteristic energy and spread, the density of states becomes

$$
g\left(\varepsilon, \varepsilon^{\prime}\right)=\frac{N_{s}}{2 \pi \sigma_{P} \sigma_{B}} \exp \left[-\frac{\left(\varepsilon-\varepsilon_{P}^{0}\right)^{2}}{2 \sigma_{P}^{2}}-\frac{\left(\varepsilon^{\prime}-\varepsilon_{B}^{0}\right)^{2}}{2 \sigma_{B}^{2}}\right] .
$$

The statistical functions are obtained from the product of terms as Eq. (11) for each energy level. The number of polarons is described by the expression

$$
n_{P}=\int_{-\infty}^{+\infty} g\left(\varepsilon, \varepsilon^{\prime}\right) f_{P}\left(\mu, \varepsilon, \varepsilon^{\prime}\right) d \varepsilon d \varepsilon^{\prime},
$$

where $f_{P}\left(\mu, \varepsilon, \varepsilon^{\prime}\right)$ is given in Eq. (15). For $B$, we get

$$
n_{B}=\int_{-\infty}^{+\infty} g\left(\varepsilon, \varepsilon^{\prime}\right) f_{B}\left(\mu, \varepsilon, \varepsilon^{\prime}\right) d \varepsilon d \varepsilon^{\prime},
$$

where $f_{B}\left(\mu, \varepsilon, \varepsilon^{\prime}\right)$ is the function in Eq. (16).
The chemical capacitances take the forms

$$
\begin{aligned}
& C_{\mu}^{P}=-e^{2} \int_{-\infty}^{+\infty} g\left(\varepsilon, \varepsilon^{\prime}\right) \frac{d f_{P}}{d \mu}\left(\mu, \varepsilon, \varepsilon^{\prime}\right) d \varepsilon, \\
& C_{\mu}^{B}=-e^{2} \int_{-\infty}^{+\infty} g\left(\varepsilon, \varepsilon^{\prime}\right) \frac{d f_{B}}{d \mu}\left(\mu, \varepsilon, \varepsilon^{\prime}\right) d \varepsilon .
\end{aligned}
$$

The results of the Gaussian distribution for both $P$ and $B$ are illustrated in Figs. 5-7. Note that the formation of two well resolved peaks, as in Fig. 3, is precluded by the distribution of energy levels of each species, $P$ and $B$.

\section{EXPERIMENTAL CONDITIONS}

Polypyrrole films were synthesized galvanostatically on different substrates, viz. indium-doped tin oxide ITO (Delta Technologies) (15 $\Omega / \square$ of surface resistance) and a Pt sheet $\left(\approx 1 \mathrm{~cm}^{2}\right.$ of active area in both cases), using acetonitrile (Baker, high purity grade), with $2 \%$ of water content, as solvent, $0.1 \mathrm{M} \mathrm{LiClO}_{4}$ (Aldrich, $>95 \%$ content) as electrolyte, and $0.1 \mathrm{M}$ pyrrole (Merck) previously distilled as monomer. Constant current densities of $0.2 \mathrm{~mA} \mathrm{~cm}^{-2}$ (ITO) and $0.4 \mathrm{~mA} \mathrm{~cm}^{-2}(\mathrm{Pt})$ were employed yielding after a sharp maximum a constant voltage near $0.75 \mathrm{~V}$ versus $\mathrm{Ag} / \mathrm{AgCl}$ reference electrode at room temperature. Polypyrrole films were prepared by using polymerization charges within the range of $60-120 \mathrm{mC} \mathrm{cm}^{-2}$ which yielded films of estimated thickness $\sim 120-240 \mathrm{~nm}$, assuming that thickness is proportional to electropolymerization charge (approximately 2 $\left.\mu \mathrm{m} \mathrm{C}^{-1} \mathrm{~cm}^{2}\right){ }^{29}$ Then films were removed from the solution and washed in distilled water, dried in air and transferred to a solution of $0.1 \mathrm{M} \mathrm{LiClO}_{4}$ in propylene carbonate (Merck,
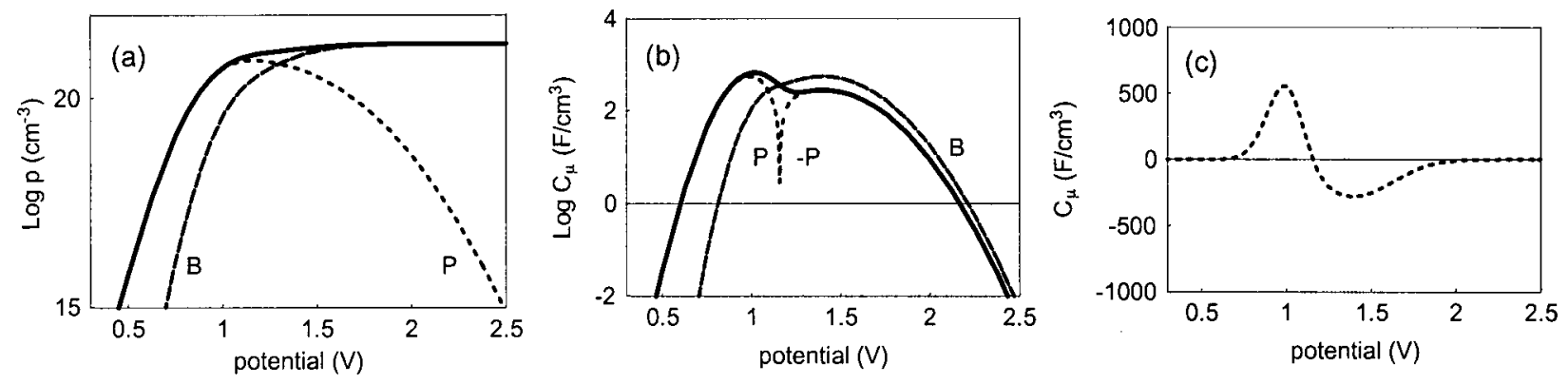

FIG. 7. Same as Fig. 5 for a Gaussian distribution of $P$ and $B$ with mean values with energies $\varepsilon_{P}=1.0 \mathrm{eV}$ and $\varepsilon_{B}=2.4 \mathrm{eV}$ and variances $\sigma_{P}=0.1 \mathrm{eV}, \sigma_{B}$ $=0.2 \mathrm{eV}$. 

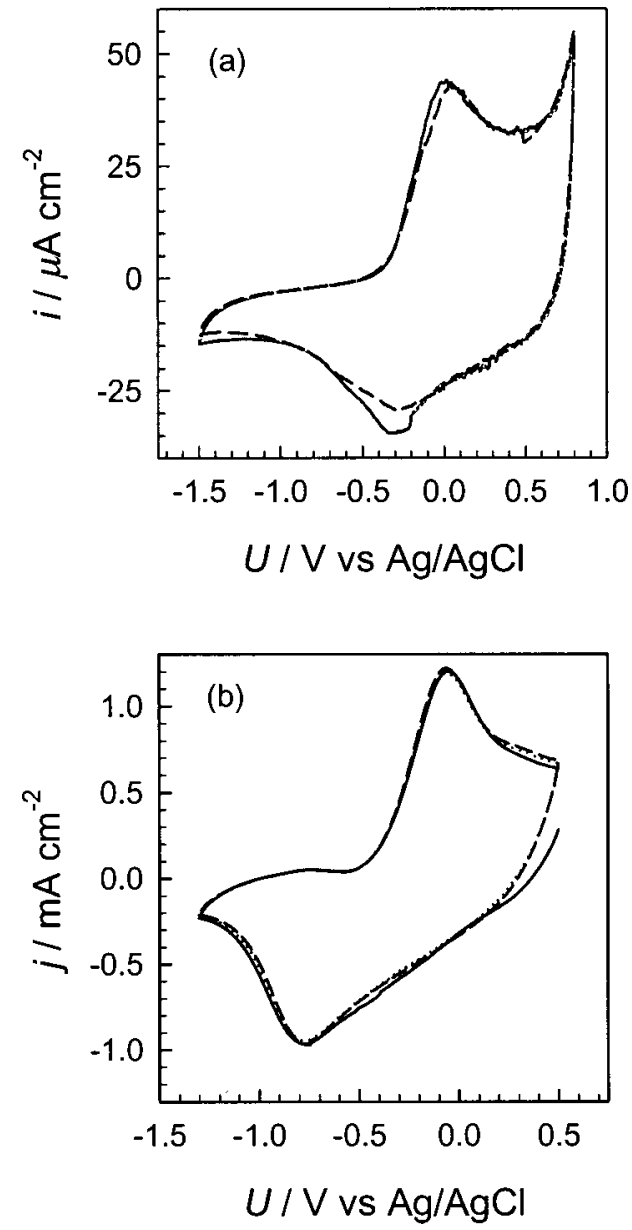

FIG. 8. Stable cyclic voltammograms of polypyrrole films (current per unit film area) in a solution of $0.1 \mathrm{M} \mathrm{LiClO}_{4}$ in propylene carbonate. (a) Onto ITO substrate and $60 \mathrm{mC} \mathrm{cm}^{-2}$ of polymerization charge. Scan rate was 10 $\mathrm{mV} \mathrm{s}^{-1}$. (b) Onto Pt substrate and $120 \mathrm{mC} \mathrm{cm}^{-2}$ of polymerization charge. Scan rate was $50 \mathrm{mV} \mathrm{s}^{-1}$.

$>99 \%$ content) in which electrochemical experiments were performed. Acetonytrile, lithium perclorate, and propylene carbonate were used as received.

The polymer films deposited onto ITO and Pt substrates were the working electrodes, Pt sheet was used as counter electrode, and $\mathrm{Ag} / \mathrm{AgCl}$ electrode as reference. For the electrochemical experiments, the solutions were deoxygenated by dry nitrogen flow through the solution maintained $30 \mathrm{~min}$ prior and over the experiments. All the experiments were performed at $25^{\circ} \mathrm{C}$. Cyclic voltammetry was performed using a potenciostat-galvanostat AutoLab PGSTAT30 equipment.

\section{RESULTS AND DISCUSSION}

Reproducible voltammetric behavior of the polypyrrole films was attained after the first $\sim 5$ scans. Some examples of this stable response are shown in Fig. 8 for films deposited on different substrates. In all cases CV's present similar characteristics: a rather broadened oxidation peak appears during the positive sweep at about $0.0 \mathrm{~V}$ versus $\mathrm{Ag} / \mathrm{AgCl}$ for scan rates within the range $10-50 \mathrm{mV} \mathrm{s}^{-1}$, followed by a current plateau at more positive potentials. For thin films and low scan rates (usual experimental conditions to preclude kinetic
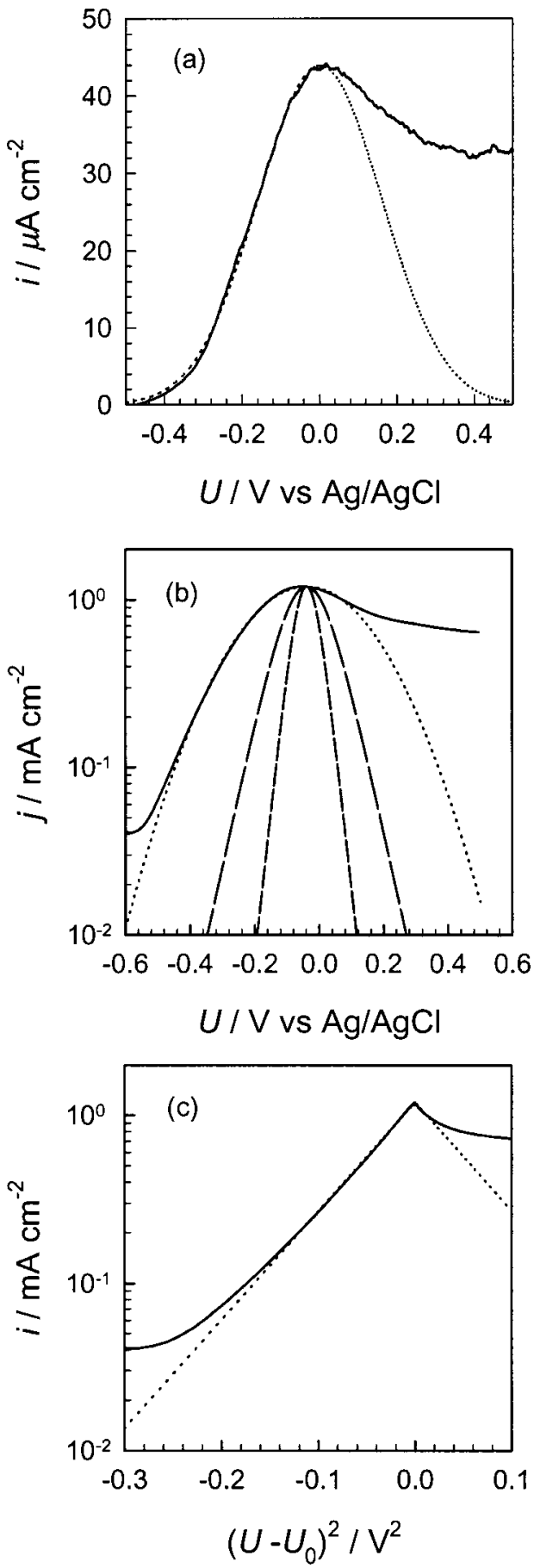

FIG. 9. (a) Comparison between CV response for potentials near the oxidation peak of polypyrrole films onto ITO substrate and $60 \mathrm{mC} \mathrm{cm}^{-2}$ of polymerization charge (continuous) and fit (dotted) using a Gaussian distribution of states $\left(\sigma=160 \mathrm{meV}, \varepsilon^{0}=1.0 \mathrm{meV}\right.$, and $\left.N_{s}=1.8 \times 10^{21} \mathrm{~cm}^{-3}\right)$. (b) Comparison between $\mathrm{CV}$ response (log current) for potentials near the oxidation peak of polypyrrole films onto $\mathrm{Pt}$ substrate and $120 \mathrm{mC} \mathrm{cm}^{-2}$ of polymerization charge (continuous) and fit (dotted) using a Gaussian distribution of states $\left(\sigma=183 \mathrm{meV}, \varepsilon^{0}=-38 \mathrm{meV}\right.$, and $\left.N_{s}=5.7 \times 10^{21} \mathrm{~cm}^{-3}\right)$. Dashed lines correspond to nernstian behavior for one (short, $60 \mathrm{mV} /$ decade) and two (long, $120 \mathrm{mV} /$ decade) species involved in the thermodynamics of doping. (c) Same data as (a) highlighting the quadratic dependence predicted by Eqs. (25) and (28), $\ln j \propto\left[\left(e U-\varepsilon^{0}\right) / k_{B} T\right]^{2}$.

limitations) the registered current is directly connected to the chemical capacitance of the polymer film as Eq. (3) because the potential applied $U$ is very close to that governing the charging process. 
As previously commented, this work is focused to the particular pattern exhibited by CV's experiments for potentials in the vicinity of the oxidation peak. Figure 9 shows CV characteristic responses of two polypyrrole films deposited onto different electrodes and the comparison to different models. We note first that the peaks are too broad to be interpreted in terms of simple nernstian statistics. The width at half heigth of the oxidation peaks $(\sim 300 \mathrm{mV}$ calculated from the anodic part of such peaks) is always quite high as compared to the nernstian widths of 90.6 or $181.2 \mathrm{mV}$ for one and two species, respectively. Moreover, the current asymptotas predicted by these models yield linear relationships of the form $\ln j \propto(e U-\varepsilon) / m k_{B} T$, in which $m$ relates to the number of species involved, as discussed in Sec. II. By examining Fig. 9(b) one can realize that the slope of the anodic part of the oxidation peak (in log-linear representation) is not constant, but decreases monotonically as the peak potential is reached. These are strong indications that the nernstian models [shown in Fig. 9(b) for comparison] are unable to explain the main features of the observed oxidation process.

In contrast, using a Gaussian distribution of sites energies we obtain readily the observed features. Indeed, the theoretical plot in Fig. 4 shows that reasonable distributions of half width between 100 and $200 \mathrm{meV}$ provide very broad oxidation peaks. In addition, the current asymptotas in Fig. 4 are not linear due to the parabollic shape implied by the Gaussian distribution.

We therefore consider the fit of the measured peaks to the Gaussian distribution. As observed in Fig. 9(a), for the polypyrrole film deposited on ITO, excellent fit is obtained for the anodic part of the oxidation peak while the cathodic part is dominated by a current plateau. Figure 9(b), for the polypyrrole film on Pt, shows also the excellent fit of the Gaussian model. Values of $\sigma \approx 170 \mathrm{meV}$ and $N_{s}$ $\approx 10^{21} \mathrm{~cm}^{-3}$ have been obtained from these fits. Figure 9(b) also shows that the Gaussian provides a much better agreement than nernstian models for either one or two species. Finally, the $j(U)$ dependence predicted from the Gaussian model, Eqs. (25) and (28), $\ln j \propto\left[\left(e U-\varepsilon^{0}\right) / k_{B} T\right]^{2}$, is emphasized in Fig. 9(c). The representation with $[(e U$ $\left.\left.-\varepsilon^{0}\right) / k_{B} T\right]^{2}$ in the $x$ axis shows clearly the Gaussian form of the chemical capacitance, when the current raises above the background level in the anodic direction. In conclusion, the oxidation peak of polypyrrole (before the current plateau) is well described by the Gaussian distribution model.

We remark that CV experiments only show a welldefined oxidation peak which precludes a direct interpretation in terms of either polarons or bipolarons. Nevertheless, the formation of bipolarons is believed to be energetically favored so that it is likely that the dispersion calculated, $\sigma$ $\sim 0.2 \mathrm{eV}$, should correspond to the energy spreading of bipolaron sites. Further resolution of the electronic species (polarons and bipolarons) involved in these peaks, and their distributions of energies, requires the combined analysis of both current (CV) and spin density (spectroelectrochemical ESR) with respect to change of potential.

\section{CONCLUSIONS}

The implications of random statistical distribution functions of polarons and bipolarons in conducting polymers for electrochemical measurements in quasiequilibrium have been discussed. The chemical capacitance with respect to the potential, in log-linear representation, shows basically a linear dependence in nernstian (single energy) models and a parabollic dependence in the presence of a Gaussian distribution of site energies. These dependences provide an important tool for analyzing the distribution of polarons and bipolarons energy levels. It was found that the broad oxidation peaks usually observed in polypyrrole can be explained by a wide Gaussian distribution of energies (half width $\sigma$ $\approx 170 \mathrm{meV}$ ), attributed to bipolarons.

\section{ACKNOWLEDGMENT}

This work was supported by Ministerio de Ciencia y Tecnología under Project No. BFM2001-3640.

${ }^{1}$ C. E. D. Chidsey and R. W. Murray, J. Phys. Chem. 90, 1479 (1986).

${ }^{2}$ M. A. Vorotyntsev and J. P. Badiali, Electrochim. Acta 39, 289 (1994).

${ }^{3}$ G. Paasch, P. H. Nguyen, and A. J. Fischer, Chem. Phys. 227, 219 (1998).

${ }^{4}$ M. A. Vorotyntsev and J. Heinze, Electrochim. Acta 46, 3309 (2001).

${ }^{5}$ D. Posadas, R. M. J. Presa, and M. I. Florit, Electrochim. Acta 46, 4075 (2001).

${ }^{6}$ W. R. McKinnon and R. R. Haering, in Modern Aspects of Electrochemistry, edited by R. E. White, J. O. M. Bockris, and B. E. Conway (Plenum, New York, 1983), Vol. 15, p. 235.

${ }^{7}$ S. Sastry, Nature (London) 409, 164 (2001).

${ }^{8}$ F. Fabregat-Santiago, I. Mora-Seró, G. Garcia-Belmonte, and J. Bisquert, J. Phys. Chem. B 107, 758 (2003).

${ }^{9}$ T. Kudo and M. Hibino, Solid State Ionics 84, 65 (1996).

${ }^{10}$ M. Porto, P. Maass, M. Meyer, A. Bunde, and W. Dieterich, Phys. Rev. B 61, 6057 (2000).

${ }^{11}$ P. M. Borsenberger, L. Pautmeier, and H. Bässler, J. Chem. Phys. 94, 5447 (1991).

${ }^{12}$ H. Bässler, Phys. Status Solidi B 175, 15 (1993).

${ }^{13}$ M. Büttiker, H. Thomas, and A. Prêtre, Phys. Lett. A 180, 364 (1993).

${ }^{14}$ M. D. Levi, C. Lopez, E. Vieil, and M. A. Vorotyntsev, Electrochim. Acta 42, 757 (1997).

${ }^{15}$ J. Jamnik and J. Maier, Phys. Chem. Chem. Phys. 3, 1668 (2001).

${ }^{16}$ W. J. Albery, Z. Chen, B. R. Horrocks, A. R. Mount, P. J. Wilson, D. Bloor, A. T. Monkman, and C. M. Elliott, Faraday Discuss. Chem. Soc. 88, 247 (1989).

${ }^{17}$ S. Feldberg, J. Am. Chem. Soc. 106, 4671 (1984).

${ }^{18}$ J. C. Scott, P. Pfluger, M. T. Krounbi, and G. B. Street, Phys. Rev. B 28, 2140 (1983)

${ }^{19}$ J. L. Brédas and G. B. Street, Acc. Chem. Res. 18, 309 (1985).

${ }^{20}$ A. Saxena and J. D. Gunton, Phys. Rev. B 35, 3914 (1987).

${ }^{21}$ R. Kubo, Statistical Mechanics (North-Holland, Amsterdam, 1965).

${ }^{22}$ F. Genoud, M. Guglielmi, M. Nechtschein, E. Genies, and M. Salmon, Phys. Rev. Lett. 55, 118 (1985).

${ }^{23}$ J. Tang, R. D. Allendoerfer, and R. A. Osteryoung, J. Phys. Chem. 96, 3531 (1992).

${ }^{24} \mathrm{H}$. Reiss, Methods of Thermodynamics (Dover, New York, 1965).

${ }^{25}$ A. Zykwinska, W. Domagala, and M. Lapkowski, Electrochem. Commun. 5, 603 (2003).

${ }^{26}$ A. Dieckmann, H. Bässler, and P. M. Borsenberg, J. Chem. Phys. 99, 8136 (1993).

${ }^{27}$ Z. G. Yu, D. L. Smith, A. Saxena, R. L. Martin, and A. R. Bishop, Phys. Rev. B 63, 085202 (2001).

${ }^{28}$ D. D. C. Bradley, Synth. Met. 54, 401 (1993).

${ }^{29}$ R. A. Bull, F.-R. F. Fan, and A. J. Bard, J. Electrochem. Soc. 129, 1009 (1982) 